\title{
Pharmacokinetics of polatuzumab vedotin in combination with R/G-CHP in patients with B-cell non-Hodgkin lymphoma
}

\author{
Colby S. Shemesh ${ }^{1} \cdot$ Priya Agarwal ${ }^{1} \cdot$ Tong Lu $^{1} \cdot$ Calvin Lee $^{2} \cdot$ Randall C. Dere $^{3} \cdot$ Xiaobin Li $^{1} \cdot$ Chunze Li $^{1} \cdot \operatorname{Jin} \mathrm{Y}_{\text {. Jin }}{ }^{1}$. \\ Sandhya Girish ${ }^{1} \cdot$ Dale Miles $^{1} \cdot$ Dan Lu ${ }^{1}$
}

Received: 4 October 2019 / Accepted: 3 March 2020 / Published online: 28 March 2020

(c) The Author(s) 2020

\begin{abstract}
Purpose The phase Ib/II open-label study (NCT01992653) evaluated the antibody-drug conjugate polatuzumab vedotin (pola) plus rituximab/obinutuzumab, cyclophosphamide, doxorubicin, and prednisone (R/G-CHP) as first-line therapy for B-cell non-Hodgkin lymphoma (B-NHL). We report the pharmacokinetics (PK) and drug-drug interaction (DDI) for pola. Methods Six or eight cycles of pola $1.0-1.8 \mathrm{mg} / \mathrm{kg}$ were administered intravenously every 3 weeks (q3w) with R/G-CHP. Exposures of pola [including antibody-conjugated monomethyl auristatin E (acMMAE) and unconjugated MMAE] and R/G-CHP were assessed by non-compartmental analysis and/or descriptive statistics with cross-cycle comparisons to cycle 1 and/or after multiple cycles. Pola was evaluated as a potential victim and perpetrator of a PK drug-drug interaction with R/G-CHP. Population PK (popPK) analysis assessed the impact of prior treatment status (naïve vs. relapsed/refractory) on pola PK.

Results Pola PK was similar between treatment arms and independent of line of therapy. Pola PK was dose proportional from 1.0 to $1.8 \mathrm{mg} / \mathrm{kg}$ with R/G-CHP. Geometric mean volume of distribution and clearance of acMMAE ranged from 57.3 to $95.6 \mathrm{~mL} / \mathrm{kg}$ and 12.7 to $18.2 \mathrm{~mL} / \mathrm{kg} /$ day, respectively. acMMAE exhibited multi-exponential decay (elimination half-life 1 week). Unconjugated MMAE exhibited formation rate-limited kinetics. Exposures of pola with R/G-CHP were similar to those in the absence of CHP; exposures of R/G-CHP in the presence of pola were comparable to those in the absence of pola.

Conclusions Pola PK was well characterized with no clinically meaningful DDIs with R/G-CHP. Findings are consistent with previous studies of pola + R/G, and support pola + R/G-CHP use in previously untreated diffuse large B-cell lymphoma.
\end{abstract}

Keywords B-cell non-Hodgkin lymphoma $\cdot$ Polatuzumab vedotin · Pharmacokinetics · Combination therapy $\cdot$ Drug interactions · Phase $\mathrm{Ib} / \mathrm{II}$

Electronic supplementary material The online version of this article (https://doi.org/10.1007/s00280-020-04054-8) contains supplementary material, which is available to authorized users.

Colby S. Shemesh

shemesh.colby@gene.com

Dan Lu

lu.dan@gene.com

1 Department of Clinical Pharmacology Oncology, Genentech Inc., 1 DNA Way, South San Francisco, CA 94080, USA

2 Clinical Science, Genentech Inc., 1 DNA Way, South San Francisco, CA 94080, USA

3 Bioanalytical Science, Genentech Inc., 1 DNA Way, South San Francisco, CA 94080, USA

\section{Introduction}

B-cell non-Hodgkin lymphoma (B-NHL) is a heterogeneous set of malignancies with varying clinical outcomes. The outcomes of B-NHL vary depending on many factors including histology; while certain patients can be cured of disease, others remain refractory or relapse. The current standard of care for the majority of patients with B-NHL is immunochemotherapy; however, treatment-related morbidity and lack of efficacy remain an obstacle, especially for middle aged/older and/or frail patients [1,2]. As such, a substantial unmet medical need in patients with NHL remains, requiring evaluation of alternate and more effective approaches [3, 4].

Antibody-drug conjugates (ADCs) have become a rapidly emerging class of therapeutics, aiming to revolutionize the 
field of cancer therapy including the treatment of B-NHL [5, 6]. ADCs are composed of a monoclonal antibody (mAb) bound to a potent cytotoxic agent through a linker to provide targeted delivery to tumor cells. Attempts to treat B-cell malignancies with ADCs in a targeted setting offer several advantages over traditional chemotherapy with enhanced biologic activity [7]. Across both hematologic and solid tumors, five ADCs have been approved including: gemtuzumab ozogamicin (Mylotarg ${ }^{\mathrm{TM}}$ ), brentuximab vedotin (Adcetris $^{\mathrm{TM}}$ ), ado-trastuzumab emtansine (Kadcyla $\left.{ }^{\mathrm{TM}}\right)$, inotuzumab ozogamicin (Besponsa ${ }^{\mathrm{TM}}$ ) and polatuzumab vedotin (pola; Polivy ${ }^{\mathrm{TM}}$ ). Over 65 additional ADCs are currently in clinical evaluation targeting a wide variety of tumors [8-10].

One broad and attractive targeting option for B-NHL using an ADC is the CD79 signaling component of the $\mathrm{B}$-cell receptor, due to its exclusive expression on the B-cell lineage and malignant B cells in patients with B-NHLs [11]. Pola was recently approved by the United States Food and Drug Administration (US FDA) in combination with bendamustine and rituximab for patients with relapsed/refractory $(\mathrm{R} / \mathrm{R})$ diffuse large $\mathrm{B}$-cell lymphoma (DLBCL) after at least two prior therapies [12]. Pola is comprised of a CD79bspecific humanized monoclonal antibody that is conjugated to the cytotoxic agent monomethyl auristatin E (MMAE) via a protease-cleavable peptide linker. Pola selectively binds to the $\mathrm{CD} 79 \mathrm{~b}$ portion of the $\mathrm{B}$-cell receptor present on the surface of malignant and non-malignant $B$ cells, triggering internalization of the complex by the cell. After internalization, the linker is cleaved, resulting in the intracellular release of MMAE, which binds to tubulin to inhibit polymerization triggering tumor cell death [11]. Pola has demonstrated promising activity with combination agents in several clinical trials in patients with B-NHL and continues to be investigated in a phase III clinical trial [13-18]. Combining pola with rituximab or obinutuzumab plus cyclophosphamide, doxorubicin, and prednisone (R/G-CHP) has a manageable safety profile and promising preliminary clinical activity in newly diagnosed DLBCL [19], supporting the initiation of a phase III trial comparing pola + R-CHP to rituximab, cyclophosphamide, doxorubicin, vincristine, and prednisone (R-CHOP).

Given that pola is administered in combination with drugs from an established standard of care, a comprehensive evaluation of clinically relevant drug-drug interactions (DDI) is necessary. Due to the complexity of ADCs, understanding their pharmacokinetics (PK) and DDI can be challenging [20-23]. DDI studies are regularly conducted with smallmolecule drugs (SMDs), but few have been evaluated for large molecules such as therapeutic proteins (TPs), mAbs, and/or ADCs, which have complex elimination processes.

Labeling language around DDI for marketed ADCs is uniquely intricate, with findings based on dedicated trials, population PK studies, non-compartmental analysis, visual/graphical inspection of exposure, and/or physiologically based PK modeling approaches. European Medicines Agency and FDA recommendations for clinical pharmacology studies with ADCs are still evolving; however, sponsors are aware of the necessity to evaluate DDI potential [24, 25].

A theoretical DDI could result from a pharmacologic effect of a TP and SMD in combination, via the cell-surface target antigen, immunogenicity modulation (e.g., drugs causing immunosuppression and altering antidrug-antibody production), impacting target-mediated drug disposition (TMDD) and/or CYP450 modulation [26-28]. MMAE (the SMD and payload component of pola), doxorubicin, and cyclophosphamide are substrates of CYP3A4 isoenzymes that exhibit variable activity across patients. Concomitant medications that are inhibitors or inducers of the same metabolic isoenzymes can influence and alter the exposure of active drugs, impacting clinical outcomes and toxicity from associated therapies. An additional theoretical PK drug-disease-drug interaction risk exists with pola, rituximab, and obinutuzumab given that these agents have the potential influence of circulating B-cell count and tumor burdens that may in turn affect TMDD for these agents.

The present article describes the PK of pola in a phase $\mathrm{Ib} /$ II open-label study of pola in combination with R/G-CHP. The investigation (1) provides insight on the $\mathrm{PK}$ of pola in combination with R/G-CHP, (2) evaluates within-study comparisons of pola PK between first-line B-NHL treatment groups, (3) enables cross-study comparisons of pola PK and rituximab/obinutuzumab PK from similar clinical studies employing the same agents, and lastly (4) assesses potential DDIs involving pola when used in combination with R/GCHP. The primary objective of these analyses is to support the development of pola combination regimens as a first-line treatment for patients with B-cell NHL.

\section{Materials and methods}

\section{Study design}

This was a phase Ib/II, multicenter, open-label, dose-escalation study (GO29044; NCT01992653). Patients with B-NHL received six or eight cycles of pola $1.0-1.8 \mathrm{mg} / \mathrm{kg}+\mathrm{R} / \mathrm{G}-$ CHP (21-day cycles; R/G-CHP was given as per the standard regimen). Patients were given either six or eight cycles of treatment based on the discretion of the investigator in accordance with local institutional practice. All patients with $\operatorname{DLBCL}(N=75)$ and follicular lymphoma $(\mathrm{FL}, N=3)$ were treatment naïve, except for one patient with FL who had $\mathrm{R} / \mathrm{R}$ disease. Additional histologies represented included mantle cell lymphoma $(N=2)$ and marginal zone lymphoma $(N=1)$. The $1.8 \mathrm{mg} / \mathrm{kg}$ pola dose was selected for combination with R/G-CHP in an expansion phase for front-line 
treatment of patients with DLBCL [19]. The details of the treatment groups and study phases are summarized in Online Resource 1.

\section{Baseline characteristics of patients with DLBCL receiving $1.8 \mathrm{mg} / \mathrm{kg}$ of polatuzumab vedotin}

A total of 66 patients with DLBCL were treated with the recommended phase 2 dose of $1.8 \mathrm{mg} / \mathrm{kg}$ of polatuzumab vedotin with R/G-CHP. Median patient age (interquartile range) was $67.5(64.0-74.0)$ years, and 34 patients (52\%) were male. Eastern Cooperative Oncology Group performance status was $0-1$ in $47(71 \%)$ patients, and 2 in 19 (29\%) patients. Fifty-six patients (85\%) had stage III-IV disease. International Prognostic Index was $0-1$ in $4(6 \%)$ patients, and 2, 3, and 4-5 in 19 (29\%), 21 (32\%), and 22 (33\%) patients, respectively. Further breakdown of baseline characteristics for other B-NHL patients receiving doses of polatuzumab vedotin $<1.8 \mathrm{mg} / \mathrm{kg}$ in the study was not provided herein as this has been reported previously [19].

\section{PK sampling and analytical methods}

The PK of pola analytes (including antibody-conjugated MMAE [acMMAE], total antibody, and unconjugated MMAE) were characterized [29, 30], in addition to the PK of rituximab, obinutuzumab, cyclophosphamide, and doxorubicin. Due to the high correlation between total antibody and acMMAE (unpublished data on file), only acMMAE and unconjugated MMAE are reported (Online Resource 1). Validated methods were used to determine plasma/serum concentrations of the analytes of interest. The assays were developed and validated in accordance with industry best practices, and the performance parameters of these assays were within industry and health authority recommendations for biopharmaceutics [31-33].

Plasma concentrations of acMMAE were quantified by immunoaffinity liquid chromatography with tandem mass spectrometry (LC-MS/MS). Pola and ADC internal standard were immobilized onto the Protein A resin and the peptide linker was enzymatically cleaved to release MMAE. The released MMAE was measured by LC-MS/MS. The lower limit of quantitation (LLOQ) for the acMMAE assay was $0.359 \mathrm{ng} / \mathrm{mL}$ acMMAE $(0.50 \mathrm{nM}$ acMMAE) in human plasma. Unconjugated MMAE plasma concentrations were determined by protein-precipitation extraction with LC-MS/ MS. Samples were fortified with internal standard (deuterated MMAE) and analytes were isolated through protein precipitation. The LLOQ for the unconjugated MMAE assay was $0.0359 \mathrm{ng} / \mathrm{mL}$ MMAE (0.05 nM MMAE) in human plasma. Rituximab and obinutuzumab serum concentrations were determined via enzyme-linked immunosorbent assays. The LLOQs for the rituximab and obinutuzumab assays were $500 \mathrm{ng} / \mathrm{mL}$ and $4.05 \mathrm{ng} / \mathrm{mL}$ in human serum, respectively. Plasma concentrations of cyclophosphamide were determined by liquid extraction/LC-MS/MS, and doxorubicin by protein precipitation/LC-MS/MS. The LLOQ for the cyclophosphamide assay was $100 \mathrm{ng} / \mathrm{mL}$ in human plasma. Circulating metabolites of cyclophosphamide such as 4-hydroxycyclophosphamide were not assessed. For all assays, quality control samples were included to judge assay acceptability.

\section{Pharmacokinetic analysis}

Non-compartmental analysis (NCA) was computed using pola cycle 1 data. NCA parameters were estimated using Phoenix WinNonlin version 6.4.0.768 (Pharsight, Inc., Mountain View, CA). PK parameters included: terminal half-life $\left(t_{1 / 2}\right.$; acMMAE only), time to maximum concentration $\left(T_{\max }\right)$, maximum concentration $\left(C_{\max }\right)$, area under the concentration-time curve from time 0 to the last quantifiable time point $\left(\mathrm{AUC}_{\text {last }}\right.$; unconjugated MMAE only), AUC from time 0 to infinity $\left(\mathrm{AUC}_{\mathrm{inf}}\right.$; acMMAE only), volume of distribution $\left(V_{\mathrm{ss}}\right)$, and clearance (CL; acMMAE only). For each value, the geometric mean and $\%$ geometric coefficients of variation $(\mathrm{CV} \%)$ were calculated.

Pola was descriptively assessed as a 'perpetrator' of DDIs with cyclophosphamide and doxorubicin by comparing cyclophosphamide and doxorubicin exposure between cycle 1 , day 1 (prior to first pola dose on cycle 1 , day 2 ) and cycle 3, day 1 (after pola dosing); prednisone was not assessed in this analysis given the wide therapeutic window of steroids and low risk for pola as a perpetrator of a PK DDI for prednisone. To evaluate pola as a 'perpetrator' for DDIs with rituximab/obinutuzumab, data were compared with those for rituximab/obinutuzumab exposure from historical studies [Study BO22334 (NCT01200758): R-CHOP or rituximab, cyclophosphamide, vincristine sulfate, and prednisone (R-CVP), with intravenous $\mathrm{R}$ as first-line treatment for FL; Study BO21003 (NCT00576758): obinutuzumab for $\mathrm{R} / \mathrm{R}$ indolent B-NHL]. Additionally, pola was assessed as a DDI 'victim' of CHP by comparing pola exposure with data from previous studies as a comparator, which included Study GO27834 where pola was administered with $\mathrm{R} / \mathrm{G}$ in the absence of CHP. Patients in Study GO27834 followed the same pola PK sampling scheme in comparison to the current study. No formal statistical testing was performed.

\section{Population pharmacokinetic (popPK) analysis to assess PK in treatment-naïve versus R/R NHL patients}

A two-analyte integrated popPK model that simultaneously describes concentrations of acMMAE and unconjugated MMAE following repeated administrations of 
pola was previously developed based on data from four clinical studies of pola in B-NHL patients $(N=460)$ who received either single-agent pola, pola with $\mathrm{R} / \mathrm{G}$, pola with $\mathrm{R} / \mathrm{G}$ and bendamustine, or pola $+\mathrm{R} / \mathrm{G}-\mathrm{CHP}$ [DCS4968g (NCT01290549), GO27834 (NCT01691898), GO29365 (NCT02257567), and GO29044] [34]. A twocompartment model with a nonspecific, time-dependent linear clearance, a linear time-dependent exponentially declining clearance, and a Michaelis-Menten clearance provided a good fit of the acMMAE plasma PK profiles. All three acMMAE elimination pathways contributed to the input to the central compartment of unconjugated MMAE, which was also described by a two-compartment model. Population PK parameters, covariate effects, and interindividual variability of model parameters were estimated. The impact of clinically relevant covariates on PK exposures of each analyte were quantified to support key label claims. The details of model building, validation and covariate assessment process were presented by Lu et al. [34]. The popPK analysis was conducted via nonlinear mixed-effects modeling with Nonlinear
Mixed-Effect Modeling (NONMEM) software, version 7.3.0 (ICON Development Solutions, Ellicott City, MD) using the first-order conditional estimation method with eta-epsilon interaction.

The popPK analysis described above was applied to assess the impact of line of therapy (treatment-naïve status vs. R/R status) on pola PK. All treatment-naïve patients $(N=80)$ were from GO29044 $(N=82)$, who were all co-administered CHP, making the impact of CHP combination or line of therapy indistinguishable in the current data. Simulations of cycle 6 exposures [AUC and $C_{\max }$; pola $1.8 \mathrm{mg} / \mathrm{kg}$ every 3 weeks (q3w)] for all patients in the four studies were performed by the final population PK model based on individual empirical Bayes estimates of PK parameters using individual covariate values, except that all patients were assumed to have co-administered anti-CD20 therapy. This approach, referred to as partial covariate correction (pCC), adjusted for the potential impact of R/G combination on pola PK. The exposure estimates of pola, including acMMAE and unconjugated MMAE in patients with R/R NHL receiving
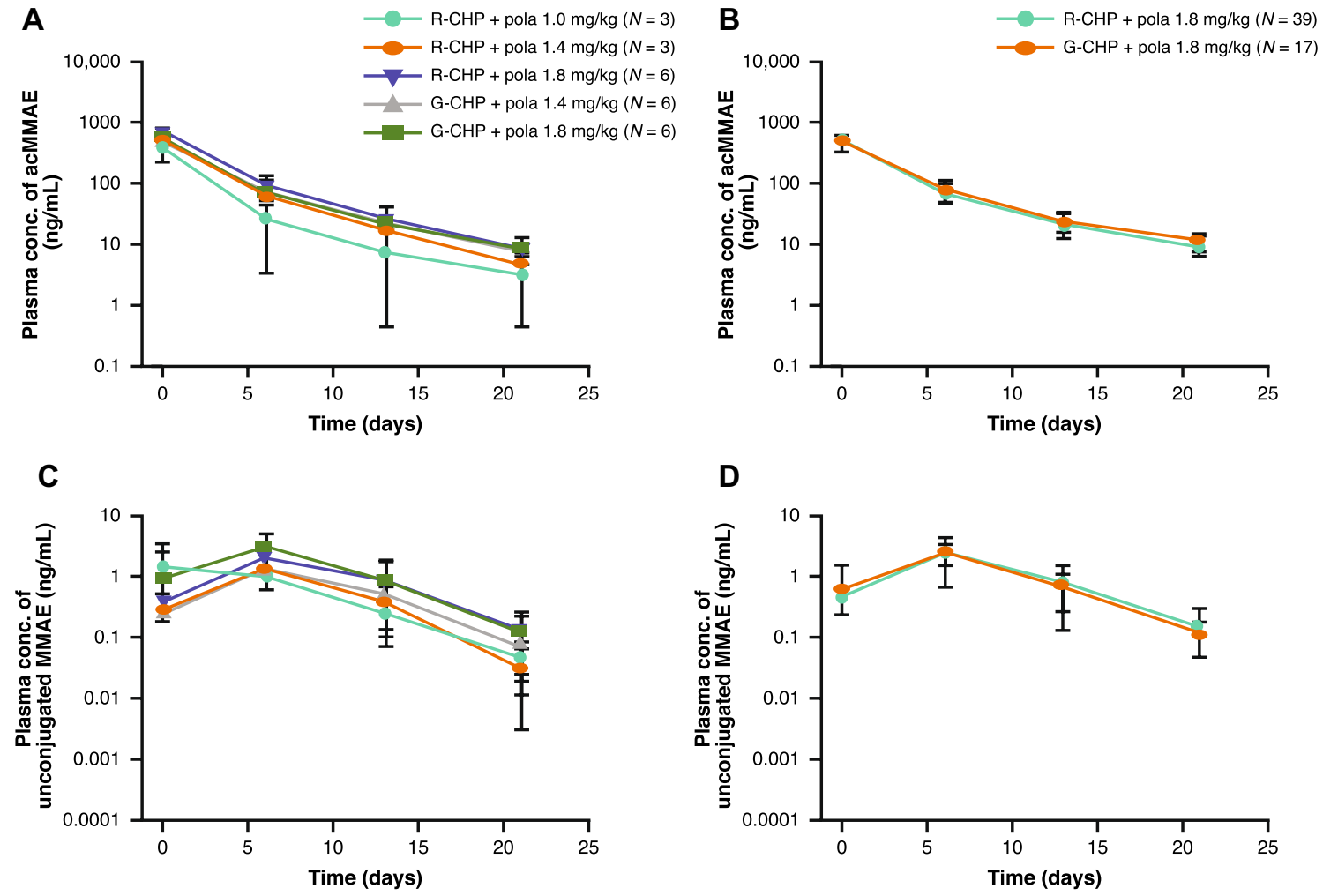

Fig. 1 Mean (SD) cycle 1 plasma/serum concentration time profiles of pola by study phase and treatment in patients receiving pola $1.0-1.8 \mathrm{mg} / \mathrm{kg}$ in combination with R-CHP, or pola $1.4-1.8 \mathrm{mg} / \mathrm{kg}$ in combination with G-CHP. Dose-escalation arms are provided in a, $\mathbf{c}$, with dose expansion arms in $\mathbf{b}, \mathbf{d}$. Pola analytes include acMMAE (a,

b) and unconjugated MMAE (c, d). acMMAE antibody-conjugated MMAE, conc concentration, G-CHP obinutuzumab, cyclophosphamide, doxorubicin, and prednisone, MMAE monomethyl auristatin $\mathrm{E}$, pola polatuzumab vedotin, $R$-CHP rituximab, cyclophosphamide, doxorubicin, and prednisone, $S D$ standard deviation 
Table 1 Cycle 1 non-compartmental pharmacokinetic parameter summary of pola

\begin{tabular}{|c|c|c|c|c|c|c|c|}
\hline Stage/population & Cohort & $N$ & $C_{\max }(\mathrm{ng} / \mathrm{mL})$ & $\mathrm{AUC}_{\text {inf }}(\mathrm{ng}$ day/mL) & $t_{1 / 2}$ (day) & $V_{\mathrm{ss}}(\mathrm{mL} / \mathrm{kg})$ & $\mathrm{CL}(\mathrm{mL} / \mathrm{kg} /$ day $)$ \\
\hline \multicolumn{8}{|c|}{ Conjugate (evaluated as acMMAE) } \\
\hline \multirow[t]{5}{*}{ Dose escalation/B-NHL } & $\mathrm{R}-\mathrm{CHP}+$ pola $1.0 \mathrm{mg} / \mathrm{kg}$ & 3 & $350(49.1)$ & $1300(1.70)^{\mathrm{a}}$ & $4.99(18.2)^{\mathrm{a}}$ & $57.7(17.3)^{\mathrm{a}}$ & $14.0(2.70)^{\mathrm{a}}$ \\
\hline & $\mathrm{R}-\mathrm{CHP}+$ pola $1.4 \mathrm{mg} / \mathrm{kg}$ & 3 & $517(33.9)$ & $1490(24.0)^{\mathrm{a}}$ & $4.82(15.0)^{\mathrm{a}}$ & $80.0(12.5)^{\mathrm{a}}$ & $17.0(24.7)^{\mathrm{a}}$ \\
\hline & $\mathrm{R}-\mathrm{CHP}$ + pola $1.8 \mathrm{mg} / \mathrm{kg}$ & 6 & $778(9.70)$ & $2580(16.0)$ & $4.75(14.2)$ & $57.3(12.6)$ & $12.7(16.1)$ \\
\hline & $\mathrm{G}-\mathrm{CHP}+$ pola $1.4 \mathrm{mg} / \mathrm{kg}$ & 5 & $534(11.2)$ & $1930(8.20)$ & $5.18(8.10)$ & $67.6(10.9)$ & $13.2(9.40)$ \\
\hline & $\mathrm{G}-\mathrm{CHP}+$ pola $1.8 \mathrm{mg} / \mathrm{kg}$ & 6 & $548(20.0)$ & $1800(27.6)$ & $4.87(10.5)$ & $85.6(23.5)$ & $18.2(27.7)$ \\
\hline \multirow[t]{2}{*}{ Expansion/DLBCL } & $\mathrm{R}-\mathrm{CHP}$ + pola $1.8 \mathrm{mg} / \mathrm{kg}$ & 36 & $503(36.5)$ & $1800(28.5)^{b}$ & $4.99(13.2)^{\mathrm{b}}$ & $91.6(33.1)^{\mathrm{b}}$ & $18.2(28.5)^{\mathrm{b}}$ \\
\hline & $\mathrm{G}-\mathrm{CHP}+$ pola $1.8 \mathrm{mg} / \mathrm{kg}$ & 17 & $513(26.6)$ & $1890(23.7)^{\mathrm{c}}$ & $5.45(14.2)^{\mathrm{c}}$ & $95.6(29.8)^{\mathrm{c}}$ & $17.3(23.4)^{\mathrm{c}}$ \\
\hline Stage/population & Cohort & & $N$ & $C_{\max }(\mathrm{ng} / \mathrm{mL})$ & $\mathrm{AUC}_{\text {last }}(\mathrm{ng} \mathrm{d}$ & $\mathrm{y} / \mathrm{mL})$ & $T_{\max }($ day $)$ \\
\hline \multicolumn{8}{|l|}{ Unconjugated MMAE } \\
\hline \multirow[t]{5}{*}{ Dose escalation/B-NHL } & \multicolumn{2}{|c|}{$\mathrm{R}-\mathrm{CHP}+$ pola $1.0 \mathrm{mg} / \mathrm{kg}$} & 3 & $1.60(107)$ & \multicolumn{2}{|l|}{$9.22(33.2)^{\mathrm{a}}$} & $5.92(0.08-6.20)$ \\
\hline & \multicolumn{2}{|c|}{$\mathrm{R}-\mathrm{CHP}+$ pola $1.4 \mathrm{mg} / \mathrm{kg}$} & 3 & $1.27(57.2)$ & \multicolumn{2}{|l|}{$10.9^{\mathrm{d}}$} & $5.95(5.80-7.08)$ \\
\hline & \multicolumn{2}{|c|}{$\mathrm{R}-\mathrm{CHP}+$ pola $1.8 \mathrm{mg} / \mathrm{kg}$} & 6 & $2.11(29.2)$ & \multicolumn{2}{|l|}{$19.5(46.6)$} & $5.98(5.87-11.9)$ \\
\hline & \multicolumn{2}{|c|}{$\mathrm{G}-\mathrm{CHP}+$ pola $1.4 \mathrm{mg} / \mathrm{kg}$} & 6 & $1.35(38.0)$ & \multicolumn{2}{|l|}{$13.4(38.3)^{\mathrm{e}}$} & $5.84(4.86-6.90)$ \\
\hline & \multicolumn{2}{|c|}{$\mathrm{G}-\mathrm{CHP}+$ pola $1.8 \mathrm{mg} / \mathrm{kg}$} & 6 & $3.00(69.7)$ & \multicolumn{2}{|l|}{$22.9(74.1)^{\mathrm{e}}$} & $5.85(0.09-5.94)$ \\
\hline \multirow[t]{2}{*}{ Expansion/DLBCL } & \multicolumn{2}{|c|}{$\mathrm{R}-\mathrm{CHP}+$ pola $1.8 \mathrm{mg} / \mathrm{kg}$} & 35 & $2.43(37.9)$ & \multicolumn{2}{|l|}{$22.6(40.4)^{\mathrm{f}}$} & $5.90(2.87-6.99)$ \\
\hline & \multicolumn{2}{|c|}{$\mathrm{G}-\mathrm{CHP}+$ pola $1.8 \mathrm{mg} / \mathrm{kg}$} & 14 & $2.44(60.0)$ & \multicolumn{2}{|l|}{$20.4(62.8)^{\mathrm{g}}$} & $5.35(0.09-6.02)$ \\
\hline
\end{tabular}

All values are geometric mean (\% geo CV), except for $T_{\max }$ (median [range])

acMMAE antibody-conjugated MMAE, $A U C_{i n f}$ area under the concentration-time curve from 0 to infinity, $A U C_{\text {last }}$ area under the concentrationtime curve from 0 until the last measurable time point, $B$-NHL B-cell non-Hodgkin lymphoma, $C L$ clearance, $C_{\max }$ maximum concentration, $D L B C L$ diffuse large B-cell lymphoma, $G-C H P$ obinutuzumab, cyclophosphamide, doxorubicin, and prednisone, $M M A E$ monomethyl auristatin E, pola polatuzumab vedotin, $R$-CHP rituximab, cyclophosphamide, doxorubicin, and prednisone, $t_{1 / 2}$ terminal half-life, $T_{\max }$ time to maximum concentration, Vss volume of distribution

${ }^{\mathrm{a}} N=2 ;{ }^{\mathrm{b}} N=28 ;{ }^{\mathrm{c}} N=11 ;{ }^{\mathrm{d}} N=1 ;{ }^{\mathrm{e}} N=5 ;{ }^{\mathrm{f}} N=27 ;{ }^{\mathrm{g}} N=10$

pola with $\mathrm{R} / \mathrm{G}(N=380)$, were compared with those in treatment-naïve patients with B-NHL $(N=80)$ receiving pola + R/G-CHP. Geometric means and CV\% were computed and tabulated, as well as the geometric mean ratio to the reference category (R/R) and its $90 \%$ confidence interval $(\mathrm{CI})$.

\section{Results}

\section{PK of pola in patients with B-NHL in the phase Ib dose-escalation arm when given in combination with R/G-CHP}

Non-compartmental analysis and descriptive statistic results, including the mean acMMAE and unconjugated MMAE plasma concentration time profiles and PK parameters across study phase, treatment group, and dose, are provided in Figs. 1a-d and 2a-d, and Table 1. Plasma concentrations of acMMAE reached $C_{\max }$ at the end of infusion, while plasma concentrations of unconjugated MMAE increased slowly and reached peak values at approximately 6 days. Given the relatively sparse sampling of unconjugated MMAE, the observed $T_{\max }$ might not reflect the actual $T_{\max }$ (which is approximately 3 days based on Study DCS4968g [11]).

As shown in Table 1, patients with B-NHL receiving pola $1.0-1.8 \mathrm{mg} / \mathrm{kg}+\mathrm{R}-\mathrm{CHP}$ or pola $1.4-1.8 \mathrm{mg} /$ $\mathrm{kg}+\mathrm{G}-\mathrm{CHP}$ had a geometric mean cycle $1 C_{\max }$ ranging from 350 to $778 \mathrm{ng} / \mathrm{mL}(N=3-6)$ for acMMAE, and $1.27-3.00 \mathrm{ng} / \mathrm{mL}(N=3-6)$ for unconjugated MMAE. Increases in the cycle $1 C_{\max }$ of acMMAE and unconjugated MMAE were approximately dose proportional (Fig. 2a, c) over the pola dose of $1.0-1.8 \mathrm{mg} / \mathrm{kg}$.

The distribution of acMMAE was mostly restricted to the central compartment, with a geometric mean $V_{\mathrm{ss}}$ ranging $57.3-85.6 \mathrm{~mL} / \mathrm{kg}(N=2-6)$ for acMMAE. The $\mathrm{CL}$ of acMMAE was low, with a geometric mean ranging 12.7-18.2 $\mathrm{mL} / \mathrm{day} / \mathrm{kg}(N=2-6)$ (Table 1$)$. The concentration-time profile of acMMAE displayed multi-exponential decay, with a geometric mean apparent $t_{1 / 2}$ ranging 4.75-5.18 days $(N=2-6)$ (Table 1$)$. Total systemic exposure by geometric mean ranged 1300-2580 ng day/ $\mathrm{mL}(N=2-6)$ for acMMAE, and 9.22-22.9 $\mathrm{ng}$ day $/ \mathrm{mL}$ $(N=2-6)$ for unconjugated MMAE across pola $+\mathrm{R} / \mathrm{G}-$ CHP groups. Similar to observations for $C_{\max }$, increases in cycle 1 AUC of acMMAE and unconjugated MMAE were generally dose proportional (Table 1, Fig. 2b, d). 
A

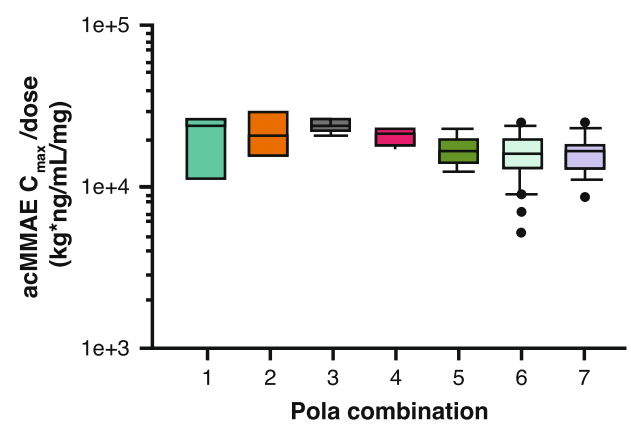

C

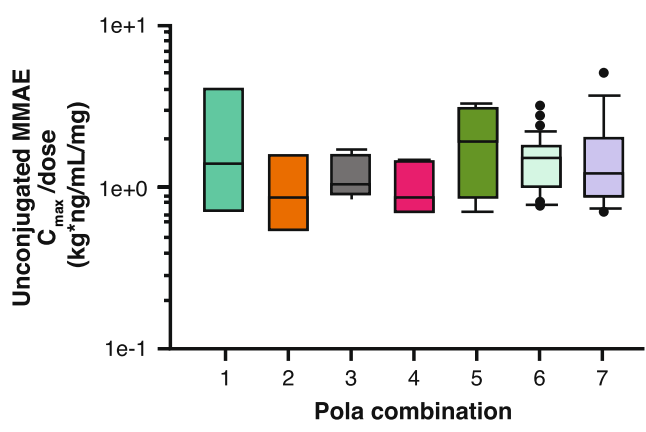

B

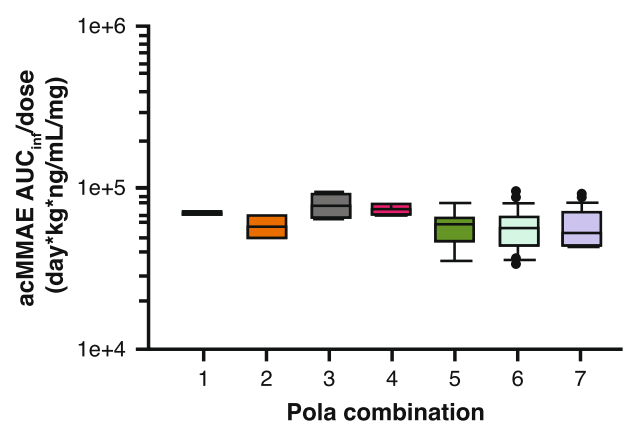

D

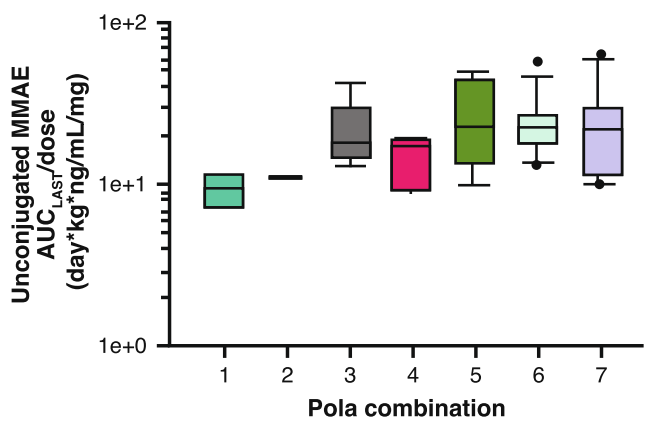

$\mathrm{R}-\mathrm{CHP}+$ pola $1.0 \mathrm{mg} / \mathrm{kg} \mathrm{ESC}(\mathrm{N}=3)$

$\mathrm{R}-\mathrm{CHP}+$ pola $1.4 \mathrm{mg} / \mathrm{kg} \mathrm{ESC}(\mathrm{N}=3)$

$\mathrm{R}-\mathrm{CHP}$ + pola $1.8 \mathrm{mg} / \mathrm{kg} \mathrm{ESC}(N=6)$

$\mathrm{G}-\mathrm{CHP}$ + pola $1.4 \mathrm{mg} / \mathrm{kg}$ ESC $(N=6)$

$\mathrm{G}-\mathrm{CHP}+$ pola $1.8 \mathrm{mg} / \mathrm{kg} \operatorname{ESC}(N=6)$

$\mathrm{R}-\mathrm{CHP}+$ pola $1.8 \mathrm{mg} / \mathrm{kg} \operatorname{EXP}(N=36)$

$\mathrm{G}-\mathrm{CHP}+$ pola $1.8 \mathrm{mg} / \mathrm{kg} \operatorname{EXP}(N=17)$

Fig. 2 Comparison of dose-normalized exposure of pola by $C_{\max }(\mathbf{a}$, c) or AUC $(\mathbf{b}, \mathbf{d})$ within cycle 1 across dose-escalation and expansion arms in patients with B-NHL or DLBCL receiving pola $1.0-1.8 \mathrm{mg} /$ $\mathrm{kg}+\mathrm{R}-\mathrm{CHP}$ or pola $1.4-1.8 \mathrm{mg} / \mathrm{kg}+\mathrm{G}-\mathrm{CHP}$. Pola analytes include acMMAE $(\mathbf{a}, \mathbf{b})$ and unconjugated $\operatorname{MMAE}(\mathbf{c}, \mathbf{d})$. Vertical boxplots include the median, 10th, 25th, 75th, and 90th percentiles as vertical boxes with error bars for each respective cohort and outliers (black bulleted circles) plotted as single point. acMMAE antibody-conju-

The systemic exposure of unconjugated MMAE was low relative to acMMAE. At the $1.8 \mathrm{mg} / \mathrm{kg}$ pola dose, geometric mean values for unconjugated MMAE $C_{\max }$ and AUC were $<2 \%$ of those for acMMAE. After three cycles of pola, plasma pre-dose concentrations of acMMAE were 2.00- to 3.71-fold higher than those seen after the first dose, indicative of accumulation with repeated dosing. However, upon repeated administration of pola, pre-dose concentrations of unconjugated MMAE remained low, and no accumulation of unconjugated MMAE was observed with the $\mathrm{q} 3 \mathrm{w}$ regimen.

\section{PK of pola in patients with DLBCL in the phase II expansion arm when given in combination with R/G-CHP}

The PK characteristics of pola in patients with DLBCL in the phase II expansion arms receiving pola $1.8 \mathrm{mg} /$ $\mathrm{kg}+\mathrm{R} / \mathrm{G}-\mathrm{CHP}$ were generally similar to those in patients gated MMAE, $A U C_{i n f}$ area under the concentration-time curve from 0 to infinity, $A U C_{\text {last }}$ area under the concentration-time curve from 0 until the last measurable time point, $B-N H L$ B-cell non-Hodgkin lymphoma, $C_{\max }$ maximum concentration, $D L B C L$ diffuse large B-cell lymphoma, ESC dose-escalation phase, EXP expansion phase, $G$-CHP obinutuzumab, cyclophosphamide, doxorubicin, and prednisone, MMAE monomethyl auristatin E, pola polatuzumab vedotin, $R$-CHP rituximab, cyclophosphamide, doxorubicin, and prednisone

with mixed subtype B-NHL in the phase Ib dose-escalation study at the same dose level. They were also similar between the R-CHP and G-CHP treatment groups (Fig. 1a-d).

Across expansion arms in patients receiving pola $1.8 \mathrm{mg} / \mathrm{kg}+\mathrm{R} / \mathrm{G}-\mathrm{CHP}$, the geometric mean cycle 1 $C_{\max }$ ranged $503-515 \mathrm{ng} / \mathrm{mL}(N=17-36)$ for acMMAE, and 2.43-2.44 ng/mL $(N=14-35)$ for unconjugated MMAE, while the geometric mean $\mathrm{AUC}_{\text {inf }}$ ranged 1800-1890 ng day/mL $(N=11-28)$ for acMMAE, and the geometric mean $\mathrm{AUC}_{\text {last }}$ was 20.4-22.6 ng day/mL $(N=10-27)$ for unconjugated MMAE (Table 1). Pre-dose concentrations of unconjugated MMAE remained low, with no accumulation of unconjugated MMAE observed with the $\mathrm{q} 3 \mathrm{w}$ regimen. 
Table 2 Assessment of CHP as a perpetrator of a PK drug-drug interaction with $1.8 \mathrm{mg} / \mathrm{kg}$ of pola as a victim based on cycle 1 non-compartmental analysis results

\begin{tabular}{|c|c|c|c|c|c|c|}
\hline Analyte & Parameter & $N$ & $\begin{array}{l}\text { GO29044 } \\
\text { (pola + R-CHP) } \\
\text { DLBCL }\end{array}$ & $N$ & $\begin{array}{l}\mathrm{GO} 27834 \\
(\text { pola }+\mathrm{R}) \\
\mathrm{FL}\end{array}$ & GMR (90\% CI) \\
\hline \multirow[t]{2}{*}{ acMMAE } & $C_{\max }(\mathrm{ng} / \mathrm{mL})$ & 36 & $503(36.4)$ & 17 & $780(14.4)$ & $0.646(0.576-0.724)$ \\
\hline & $\mathrm{AUC}_{\text {inf }}(\mathrm{ng}$ day/mL) & 28 & $1800(28.5)$ & 15 & $2530(25.9)$ & $0.711(0.616-0.820)$ \\
\hline \multirow[t]{2}{*}{ Unconjugated MMAE } & $C_{\max }(\mathrm{ng} / \mathrm{mL})$ & 35 & $2.43(37.9)$ & 20 & $1.75(54.3)$ & $1.39(1.11-1.73)$ \\
\hline & $\mathrm{AUC}_{\text {last }}(\mathrm{ng}$ day/mL) & 27 & $22.6(40.4)$ & 20 & $15.8(50.1)$ & $1.43(1.15-1.78)$ \\
\hline Analyte & Parameter & $N$ & $\begin{array}{l}\text { GO29044 } \\
\text { (pola + G-CHP) } \\
\text { DLBCL }\end{array}$ & $N$ & $\begin{array}{l}\text { GO27834 } \\
(\text { pola + G) } \\
\text { DLBCL }\end{array}$ & GMR (90\% CI) \\
\hline \multirow[t]{2}{*}{ acMMAE } & $C_{\max }(\mathrm{ng} / \mathrm{mL})$ & 17 & $513(26.6)$ & 33 & $694(22.7)$ & $0.739(0.651-0.839)$ \\
\hline & $\mathrm{AUC}_{\text {inf }}(\mathrm{ng}$ day/mL) & 11 & $1890(23.7)$ & 26 & $2350(28.4)$ & $0.805(0.691-0.938)$ \\
\hline \multirow[t]{2}{*}{ Unconjugated MMAE } & $C_{\max }(\mathrm{ng} / \mathrm{mL})$ & 14 & $2.44(60.0)$ & 40 & $2.68(81.1)$ & $0.911(0.664-1.25)$ \\
\hline & $\mathrm{AUC}_{\text {last }}(\mathrm{ng}$ day/mL) & 10 & $20.4(62.8)$ & 40 & $22.5(70.7)$ & $0.907(0.629-1.31)$ \\
\hline
\end{tabular}

All values are geometric mean (\% geo CV), except for GMR

acMMAE antibody-conjugated MMAE, $A U C_{\text {inf }}$ area under the concentration-time curve from 0 to infinity, $A U C_{\text {last }}$ area under the concentration-time curve from 0 until the last measurable time point, $C H P$ cyclophosphamide, doxorubicin, and prednisone, $C I$ confidence interval, $C_{m a x}$ maximum concentration, $C V$ coefficient of variation, $D L B C L$ diffuse large B-cell lymphoma, $F L$ follicular lymphoma, $G$ obinutuzumab, $G$ - $C H P$ obinutuzumab, cyclophosphamide, doxorubicin, and prednisone, $G M R$ geometric mean ratio, $M M A E$ monomethyl auristatin E, $P K$ pharmacokinetic, pola polatuzumab vedotin, $R$ rituximab, $R-C H P$ rituximab, cyclophosphamide, doxorubicin, and prednisone

\section{Potential PK drug-drug interaction of pola and R/G-CHP}

The potential for PK DDI between CHP and pola was evaluated using exposure comparisons between patients receiving pola + R/G-CHP and those with R/R DLBCL or FL receiving pola with $\mathrm{R} / \mathrm{G}$ in a phase II study [17, 35]. Exposure comparisons included cycle $1 C_{\max }$ and AUC of each pola analyte including acMMAE and unconjugated MMAE. Pola was not a victim of a drug-drug interaction with CHP as there were no clinically meaningful differences between geometric mean exposures of acMMAE and unconjugated MMAE after the first $1.8-\mathrm{mg} / \mathrm{kg}$ dose of pola $+\mathrm{R} / \mathrm{G}-\mathrm{CHP}$ vs. pola $+R / G$ (Table 2), as summarized below. It is worth noting that, as mentioned in the method section for popPK analysis, the effect of CHP combination is indistinguishable from line of therapy (treatment-naïve vs. R/R status).

For exposure assessments of pola $+\mathrm{R}-\mathrm{CHP}$ compared with pola combined with rituximab (without $\mathrm{CHP}$ ), a direct comparison in patients of the same B-NHL type was not possible. However, given DLBCL and FL patients have generally similar PK for pola, a cross-study comparison of available data was conducted. Within cycle 1, exposure of pola in patients with treatment-naïve DLBCL receiving pola + R-CHP showed a geometric mean ratio (GMR) for AUC of 0.711 (90\% CI 0.616-0.820) for acMMAE and 1.43 (90\% CI 1.15-1.78) for unconjugated MMAE when compared with R/R FL patients receiving pola with rituximab (in the absence of CHP); this is likely reflective of cross-study variations and within the variability of each analyte ( $\sim 30 \%$ for acMMAE, and $\sim 60 \%$ for unconjugated MMAE) (Table 2).

For the obinutuzumab-containing cohorts, systemic exposure comparisons in cycle 1 (AUC) indicated that the addition of CHP to pola and obinutuzumab did not appear to substantially impact the PK of pola. The GMR for cycle 1 AUC comparisons (for DLBCL in Study GO29044 vs. DLBCL in Study GO27834) was 0.805 (90\% CI 0.691-0.938) for acMMAE, and 0.907 (90\% CI 0.629-1.31) for unconjugated MMAE, for pola + G-CHP compared with pola + obinutuzumab only (Table 2). These differences were within the PK variability of each analyte and could also be attributed to differences in patient characteristics, and, given the acceptable safety profiles of all treatment arms, were not considered clinically meaningful.

\section{Pola PK in treatment-naïve versus R/R NHL using a population $\mathrm{PK}$ approach}

All of the treatment-naïve patients in the analysis were from the current study (GO29044), while R/R patients were pooled from several other studies for comparison. Based on the integrated acMMAE-MMAE population PK model using a pCC approach, patients who were treatment naïve had approximately $20 \%$ higher central $V_{\mathrm{ss}}$ for acMMAE compared with patients with $\mathrm{R} / \mathrm{R}$ disease. As shown in Table 3, treatment-naïve patients also had lower acMMAE exposures (by $8 \%$ for AUC, and $15 \%$ for $C_{\max }$ ) and lower 
Table 3 Population PK comparison of pola exposure $(1.8 \mathrm{mg} / \mathrm{kg} \mathrm{q} 3 \mathrm{w})$ at cycle 6 by treatment status (treatmentnaïve vs. relapsed or refractory)

\begin{tabular}{lllll}
\hline Pola analyte & Parameter & Geometric mean $(\% \mathrm{CV})$ & GMR (90\% CI) \\
\cline { 3 - 4 } & & $\mathrm{R} / \mathrm{R}(N=380)$ & $\begin{array}{l}\text { Treatment-naïve } \\
(N=80)\end{array}$ \\
\hline acMMAE & $\mathrm{AUC}, \mathrm{ng}$ day/mL & $2950(21)$ & $2720(15)$ & $0.92(0.89-0.96)$ \\
& $C_{\max }, \mathrm{ng} / \mathrm{mL}$ & $734(15)$ & $621(17)$ & $0.85(0.82-0.88)$ \\
Unconjugated MMAE & $\mathrm{AUC}, \mathrm{ng}$ day $/ \mathrm{mL}$ & $20.9(50)$ & $15.5(42)$ & $0.74(0.68-0.80)$ \\
& $C_{\max }, \mathrm{ng} / \mathrm{mL}$ & $1.93(46)$ & $1.42(37)$ & $0.74(0.68-0.80)$ \\
\hline
\end{tabular}

All treatment-naïve B-NHL patients $(N=80)$ were from the current Study GO29044, who received pola coadministered with CHP chemotherapy. Relapsed/refractory B-NHL patients $(N=380)$ were from Studies DCS4968g (NCT01290549), GO27834 (NCT01691898), and GO29365 (NCT02257567), which included patients who received either single-agent pola, pola with $\mathrm{R} / \mathrm{G}$, or pola with $\mathrm{R} / \mathrm{G}$ and bendamustine

acMMAE antibody-conjugated MMAE, $A U C$ area under the curve, B-NHL B-cell non-Hodgkin lymphoma, $C H P$ cyclophosphamide, doxorubicin, and prednisone, $C I$ confidence interval, $C_{\max }$ maximum concentration, $C V$ coefficient of variation, $G$ obinutuzumab, $G M R$ geometric mean ratio, MMAE, monomethyl auristatin E, $P K$ pharmacokinetic, pola polatuzumab vedotin, $q 3 w$ every 3 weeks, $R$ rituximab, $R / R$ relapsed/refractory

Table 4 Assessment of $1.8 \mathrm{mg} / \mathrm{kg}$ of pola as a perpetrator of a PK drug-drug interaction with cyclophosphamide, doxorubicin, rituximab, and obinutuzumab as a victim based on descriptive statistics of exposure comparisons

\begin{tabular}{|c|c|c|c|c|c|c|c|}
\hline DDI victim & $\mathrm{Tx}$ & Parameter & $N$ & $\begin{array}{l}\text { GO29044 (C1D1) } \\
\text { Before pola dosing }\end{array}$ & $N$ & $\begin{array}{l}\text { GO29044 (C3D1) } \\
\text { After pola dosing }\end{array}$ & GMR (90\% CI) \\
\hline \multirow[t]{2}{*}{ Cyclophosphamide $(\mu \mathrm{g} / \mathrm{mL})$} & R-CHP & $\mathrm{C}_{23 \mathrm{~h}}$ & 25 & $2.64(56.2)$ & 19 & $2.67(74.8)$ & $1.01(0.737-1.38)$ \\
\hline & G-CHP & $\mathrm{C}_{23 \mathrm{~h}}$ & 14 & $3.00(52.4)$ & 14 & $2.83(50.1)$ & $0.943(0.691-1.29)$ \\
\hline \multirow[t]{2}{*}{ Doxorubicin (ng/mL) } & R-CHP & $\mathrm{C}_{24 \mathrm{~h}}$ & 25 & $8.79(29.1)$ & 20 & $8.43(25.8)$ & $0.959(0.838-1.10)$ \\
\hline & G-CHP & $\mathrm{C}_{24 \mathrm{~h}}$ & 12 & $9.44(60.6)$ & 14 & $8.94(21.3)$ & $0.947(0.701-1.28)$ \\
\hline DDI victim & $\mathrm{Tx}$ & Parameter & $n$ & $\begin{array}{l}\text { BO22334 } \\
\text { R-CHOP }\end{array}$ & $n$ & $\begin{array}{l}\text { GO29044 } \\
\text { Pola + R-CHP }\end{array}$ & GMR (90\% CI) \\
\hline Rituximab ( $\mu \mathrm{g} / \mathrm{mL})$ & R-CHP & $\mathrm{C} 4$ pre-dose & 189 & $45.0(111)$ & 28 & $66.3(36.2)$ & $1.47(1.26-1.72)$ \\
\hline DDI victim & $\mathrm{Tx}$ & Parameter & $n$ & $\begin{array}{l}\mathrm{BO} 21003 \\
\mathrm{G}\end{array}$ & $n$ & $\begin{array}{l}\text { GO29044 } \\
\text { Pola + G-CHP }\end{array}$ & GMR (90\% CI) \\
\hline Obinutuzumab $(\mu \mathrm{g} / \mathrm{mL})$ & G-CHP & C2 pre-dose & 74 & $378(60.5)$ & 15 & $266(71.0)$ & $0.703(0.517-0.955)$ \\
\hline
\end{tabular}

All values are geometric mean (\% geo CV), except for GMR. GO29044=1L DLBCL; BO21003=R/R indolent B-cell NHL; and BO22334=1L FL

$1 L$ first line, $C$ cycle, $C_{23 h}$ concentration $23 \mathrm{~h}$ after dosing with DDI victim, $C_{24 h}$ concentration $24 \mathrm{~h}$ after dosing with DDI victim, $C I$ confidence interval, $C V$ coefficient of variation, $D$ day, $D D I$ drug-drug interaction, $D L B C L$ diffuse large B-cell lymphoma, $F L$ follicular lymphoma, $G$ obinutuzumab, $G$-CHP obinutuzumab, cyclophosphamide, doxorubicin, and prednisone, GMR geometric mean ratio, $N H L$ non-Hodgkin lymphoma, $P K$ pharmacokinetic, pola polatuzumab vedotin, $R$ - $C H O P$ rituximab, cyclophosphamide, doxorubicin, vincristine, and prednisone, $R$ - $C H P$ rituximab, cyclophosphamide, doxorubicin, and prednisone, $R / R$ relapsed/refractory, $t x$ treatment

unconjugated MMAE exposures (by $26 \%$ for both AUC and $C_{\max }$ ) than patients with $\mathrm{R} / \mathrm{R}$ disease (cycle 6), but these differences were small and within the CV\%, so were not considered clinically relevant given lower unconjugated MMAE exposures would not adversely affect safety. As mentioned in the method section, as both differences in line of therapy and disease type were present between studies, the impact of the covariate of 'treatment-naïve versus $R / R$ ' status was indistinguishable from the impact of the combination with CHP versus without CHP.

\section{PK of rituximab in combination with pola + CHP, and the potential of pola to influence the PK of rituximab}

After the first dose of rituximab $375 \mathrm{mg} / \mathrm{m}^{2}$, the geometric mean $(\mathrm{CV} \%)$ serum $C_{\max }$ reached $165(37.2) \mu \mathrm{g} / \mathrm{mL}(N=24)$, increasing to $237(18.3) \mu \mathrm{g} / \mathrm{mL}(N=28)$ by cycle 4 . The minimum concentration $\left(C_{\min }\right)$ levels of rituximab following cycle 3 (cycle 4 pre-dose) in DLBCL patients in Study GO29044 had a GMR of 1.47 (90\% CI 1.26-1.72) when compared with Study BO22334 (R-CHOP) in the absence of pola (Table 4). 
Differences observed with cross-study comparison of rituximab exposure are within the variability of rituximab observed in BO22334 (up to $111 \%$ CV). Furthermore, the impact of pola on rituximab has been formally assessed in a population PK analysis of rituximab (Roche/Genentech data on file). The model included rituximab PK data collected in the four studies of pola (DCS4968g, GO27834, GO29044, and GO29365) with or without co-administration of pola. Based on a covariate assessment with pola on rituximab PK, combination with pola was not a significant covariate of rituximab exposure.

\section{PK of obinutuzumab in combination with pola and CHP, and the potential of pola to influence the PK of obinutuzumab}

Following the first intravenous (IV) infusion of obinutuzumab $1000 \mathrm{mg}$, the geometric mean (CV\%) serum $C_{\max }$ was $346(20.4) \mu \mathrm{g} / \mathrm{mL}(N=11)$. Matching obinutuzumab dosing regimens for patients with DLBCL in Study GO29044 up to cycle 2 in patients with B-NHL in Study BO21003 allowed for a cross-study comparison of pola + G-CHP to single-agent obinutuzumab therapy based on cycle 2 pre-dose concentrations. The cycle 2 pre-dose concentration of obinutuzumab was lower when administered with pola + CHP (Study GO29044) versus administration as a monotherapy (Study BO21003); the GMR was 0.703 (90\% CI 0.517-0.955), as shown in Table 4. This difference was well within the variability of obinutuzumab (60\% CV in Study BO21003), and may reflect variability in body weight, gender, and tumor burden due to differences in patient populations between the studies (e.g., DLBCL in GO29044 vs. R/R indolent B-cell NHL in $\mathrm{BO} 21003)$.

\section{PK of cyclophosphamide and doxorubicin in combination with pola, prednisone, rituximab or obinutuzumab, and the potential of pola to influence the PK of cyclophosphamide and doxorubicin}

Cyclophosphamide and doxorubicin PK were measured in patients with DLBCL prior to and after the administration of pola. After IV infusion of the first dose of cyclophosphamide and before administration of pola, minimum plasma concentrations $\left(C_{\min }\right)$ of cyclophosphamide had a GMR of $1.01(90 \%$ CI 0.737-1.38) for R-CHP and 0.943 (90\% CI 0.691-1.29) for G-CHP compared with subsequent concomitant dosing with pola by cycle 3 . Similarly, after IV infusion of the first dose of doxorubicin and before administration of pola, $C_{\min }$ of doxorubicin had a GMR of 0.959 (90\% CI 0.838-1.10) for R-CHP and 0.947 (90\% CI 0.701-1.28) for G-CHP compared with subsequent concomitant dosing with pola by cycle 3 . These findings suggest that there is no impact of pola as a perpetrator on cyclophosphamide or doxorubicin PK (Table 3). Minimal differences in cyclophosphamide or doxorubicin PK were observed between DLBCL patients receiving either pola $+\mathrm{R}-\mathrm{CHP}$ or pola $+\mathrm{G}-\mathrm{CHP}$.

\section{Discussion}

ADCs are currently being evaluated in many different oncologic diseases, as consolidation or maintenance therapy, as single agents and in combination with other therapies, and in first-line and R/R settings [36-38]. Given the broad clinical versatility of these agents, theoretical DDI risks with ADCs related to the conjugate and cytotoxic drug exist when given in combination with another TP/SMD [28, 39-43]. Furthermore, as both ADCs and chemotherapeutic agents have narrow therapeutic windows, it is imperative that the effects of conventional drugs on the ADC, as well as the effect of the ADC on conventional drugs, are assessed [44]. A limit of 6-8 cycles of pola $1.8 \mathrm{mg} / \mathrm{kg}$ has been determined to be effective reducing the risk of peripheral neuropathy (a known adverse event [AE] with pola as a single agent) [45].

The potential impact of rituximab and obinutuzumab on the PK of pola was explored using a pooled population $\mathrm{PK}$ model analysis across multiple studies by a pCC approach assuming all patients are $\mathrm{R} / \mathrm{R}$ for treatment status (unpublished data on file). The presence of rituximab combination was identified as a significant covariate in the model, with a mild impact on steady-state (cycle 6) exposure of acMMAE (24\% higher for AUC) and moderately lower impact on steady-state exposure of unconjugated MMAE (37\% lower for AUC and $40 \%$ lower for $C_{\max }$ ), which were within the inter-individual variability of both analytes and not considered clinically meaningful based on exposure-response analysis (unpublished data on file). A similar magnitude of impact was observed for the obinutuzumab combination as demonstrated by similar exposures ( $<5 \%$ difference) compared with the rituximab combination.

The results of our investigation show that pola-related analytes have generally similar plasma/serum PK when given in combination with rituximab- and obinutuzumab-containing therapies, and between patients with mixed subtypes of B-NHL or DLBCL, and between treatment-naïve versus $\mathrm{R} / \mathrm{R}$ status. Based on the range of geometric means across treatment arms in study GO29044, acMMAE was characterized by a $V_{\text {ss }}$ mostly restricted to the central compartment (57.3-95.6 mL/kg), including a low CL (12.7-18.2 mL/kg/ day) and long $t_{1 / 2}(4.75-5.45$ days), which is consistent with prior knowledge $[14,15]$. Moderately higher acMMAE concentrations were seen in subsequent cycles with q3w dosing relative to cycle 1 , which indicated accumulation, potentially due to time-dependent decrease of clearance of acMMAE (unpublished data on file). Unconjugated MMAE maximum 
plasma concentrations were substantially lower than those for acMMAE, with an average unconjugated MMAE $C_{\max }$ and AUC of $<2 \%$ of corresponding acMMAE values. The delayed $t_{\max }$ of unconjugated MMAE suggests a formation rate-limited kinetics whereby the rate of elimination of unconjugated MMAE may be limited by its slow release from the antibody-drug conjugate. Unconjugated MMAE did not accumulate in plasma with the $\mathrm{q} 3 \mathrm{w}$ regimen.

Comparisons of cycle 1 exposures of cyclophosphamide and doxorubicin (prior to pola administration) were similar to those in cycle 3 (after pola administration), suggesting that pola does not impact the PK of cyclophosphamide or doxorubicin. The subtle differences observed in acMMAE exposure between the dose-escalation and expansion phases for patients receiving pola $+\mathrm{R}-\mathrm{CHP}$ may be related to the relatively small dose-escalation sample size, and/or disease heterogeneity across studies. Similarly, the minor trend of slightly lower acMMAE and lower/higher unconjugated MMAE exposures in the presence of CHP, observed with both population PK and NCA approaches, may be attributed to the treatment-naïve condition, CHP effect, and/or study/individual PK variability. Significant overlap in the distribution of pola exposure exists, with the magnitude of differences being within the inter-individual variability of each analyte in the current study. These observations are not unexpected given the moderate inter-individual variability in exposure seen across other ADCs [46, 47].

The overall safety profile of pola added to R/G-CHP, including serious AEs, grade 3-4 AEs and events of peripheral neuropathy [45], is manageable and largely similar to treatment with R/G-CHOP, with vincristine, in the absence of pola administration in similar patient populations $[19,48]$. Incorporation of $1.8 \mathrm{mg} / \mathrm{kg}$ of polatuzumab vedotin did not alter the safety profile described previously with R/G-CHOP in patients with DLBCL. While the AE profile in the current study varied by treatment arm, with a slightly larger incidence of grade 3-4 neutropenia and thrombocytopenia within the G-CHP arm compared to the R-CHP arm [18], exposures of acMMAE and unconjugated MMAE in both arms were similar at the $1.8-\mathrm{mg} / \mathrm{kg}$ dose level of pola. It has been shown that acMMAE is the key analyte driving both the safety and efficacy of pola [45, 49], although unconjugated MMAE is also associated with safety. In the current study, exposures of acMMAE and unconjugated MMAE did not appear to account for the slight discrepancy between the incidence of common AEs between treatment arms. Lastly, the dose intensity and need for dose modifications (dose delay/reduction, or drug discontinuation) were in line with the disease population undergoing cytotoxic therapies, suggesting that the combination of pola with immunochemotherapy was tolerable [19]. The population PK analysis described here also confirmed no clinically relevant impact of CHP combination or treatment-naïve status on the PK of either acMMAE or unconjugated MMAE.

Taken together, this report provides new insights and increases our clinical experience and understanding of pola pharmacokinetics when administered in combination with R/G-CHP, including the evaluation of the potential for DDI with the combination. Based on within-study and cross-study comparisons, there was no evidence for clinically meaningful DDIs among study treatments. The PK of pola was well characterized and consistent with expectations from previous studies employing pola, R/G, and/or CHP. No clinically meaningful differences of pola PK due to line of therapy (treatment-naïve vs. R/R status) were observed. The findings herein in addition to the favorable benefit/risk profile support the development strategy of pola + R/G-CHP as a first-line therapy in patients with B-NHL.

Acknowledgements We would like to thank the patients and their families who participated in this trial, and without whom this study would not have been possible. We also acknowledge and thank the investigators and site staff. Medical writing support was provided by Rishard Salie, Ph.D., Scientific Communication Director, Global Product Development/Medical Affairs Hematology, F. Hoffmann-La Roche Ltd, Basel, Switzerland and Angela Rogers Ph.D., Gardiner-Caldwell Communications, Macclesfield, UK.

\section{Compliance with ethical standards}

Conflict of interest This study was sponsored by Genentech, Inc and F. Hoffmann-La Roche Ltd. All study authors are employees and stockholders of Genentech, Inc and F. Hoffmann-La Roche Ltd.

Ethical approval All procedures performed in studies involving human participants were in accordance with the ethical standards of the institutional and/or national research committee and with the 1964 Helsinki declaration and its later amendments or comparable ethical standards. The first ethics committee approval was granted on 15 October 2013 from the Oncology Associates of Oregon, P.C., Willamette Valley Cancer Institute. This site granted approval for the trial in addition to other multiple ethics committees/institutional review board approvals obtained across other sites and countries of enrollment.

Informed consent Informed consent was obtained from all individual participants included in the study.

Open Access This article is licensed under a Creative Commons Attribution 4.0 International License, which permits use, sharing, adaptation, distribution and reproduction in any medium or format, as long as you give appropriate credit to the original author(s) and the source, provide a link to the Creative Commons licence, and indicate if changes were made. The images or other third party material in this article are included in the article's Creative Commons licence, unless indicated otherwise in a credit line to the material. If material is not included in the article's Creative Commons licence and your intended use is not permitted by statutory regulation or exceeds the permitted use, you will need to obtain permission directly from the copyright holder. To view a copy of this licence, visit http://creativecommons.org/licenses/by/4.0/. 


\section{References}

1. Chao MP (2013) Treatment challenges in the management of relapsed or refractory non-Hodgkin's lymphoma-novel and emerging therapies. Cancer Manag Res 5:251-269

2. Bron D, Aurer I, Andre MPE, Bonnet C, Caballero D, Falandry C, Kimby E, Soubeyran P, Zucca E, Bosly A, Coiffier B (2017) Unmet needs in the scientific approach to older patients with lymphoma. Haematologica 102:972-975

3. Polson AG, Yu SF, Elkins K, Zheng B, Clark S, Ingle GS, Slaga DS, Giere L, Du C, Tan C, Hongo JA, Gogineni A, Cole MJ, Vandlen R, Stephan JP, Young J, Chang W, Scales SJ, Ross S, Eaton D, Ebens A (2007) Antibody-drug conjugates targeted to CD79 for the treatment of non-Hodgkin lymphoma. Blood 110:616-623

4. Prichard M, Harris T, Williams ME, Densmore JJ (2009) Treatment strategies for relapsed and refractory aggressive non-Hodgkin's lymphoma. Expert Opin Pharmacother 10:983-995

5. Chu YW, Polson A (2013) Antibody-drug conjugates for the treatment of B-cell non-Hodgkin's lymphoma and leukemia. Future Oncol 9:355-368

6. Peters C, Brown S (2015) Antibody-drug conjugates as novel anticancer chemotherapeutics. Biosci Rep 35:e0225

7. Kamath AV, Iyer S (2015) Preclinical pharmacokinetic considerations for the development of antibody drug conjugates. Pharm Res 32:3470-3479

8. Lambert JM, Morris CQ (2017) Antibody-drug conjugates (ADCs) for personalized treatment of solid tumors: a review. Adv Ther 34:1015-1035

9. Beck A, Goetsch L, Dumontet C, Corvaia N (2017) Strategies and challenges for the next generation of antibody-drug conjugates. Nat Rev Drug Discov 16:315-337

10. Lambert JM, Berkenblit A (2018) Antibody-drug conjugates for cancer treatment. Annu Rev Med 69:191-207

11. Dornan D, Bennett F, Chen Y, Dennis M, Eaton D, Elkins K, French D, Go MA, Jack A, Junutula JR, Koeppen H, Lau J, McBride J, Rawstron A, Shi X, Yu N, Yu SF, Yue SF, Yue P, Zheng B, Ebens A, Polson AG (2009) Therapeutic potential of an anti-CD79b antibody-drug conjugate, anti-CD79b-vc-MMAE, for treatment of non-Hodgkin lymphoma. Blood 114:2721-2729

12. Genentech, Inc. (2019) POLIVYTM (polatuzumab vedotin-piiq) for injection, for intravenous use. https://www.accessdata.fda.gov/ drugsatfda_docs/label/2019/761121s000lbl.pdf. Accessed 4 July 2019

13. Palanca-Wessels MC, Czuczman M, Salles G, Assouline S, Sehn LH, Flinn I, Patel MR, Sangha R, Hagenbeek A, Advani R, Tilly H, Casanovas O, Press OW, Yalamanchili S, Kahn R, Dere RC, Lu D, Jones S, Jones C, Chu YW, Morschhauser F (2015) Safety and activity of the anti-CD79B antibody-drug conjugate polatuzumab vedotin in relapsed or refractory B-cell non-Hodgkin lymphoma and chronic lymphocytic leukemia: a phase 1 study. Lancet Oncol 16:704-715

14. Morschhauser F, Flinn I, Advani RH, Diefenbach CS, Kolibaba K, Press OW, Sehn LH, Chen AI, Salles G, Tilly H, Cheson BD, Assouline S, Dreyling M, Hagenbeek A, Zinzani PL, Yalamanchili S, Lu D, Jones C, Jones S, Chu YW, Sharman JP (2014) Updated results of a phase II randomized study (ROMULUS) of polatuzumab vedotin or pinatuzumab vedotin plus rituximab in patients with relapsed/refractory non-Hodgkin lymphoma. Blood 124:4457

15. Herrera AF, Molina A (2018) Investigational antibody-drug conjugates for treatment of B-linear malignancies. Clin Lymphoma Myeloma Leuk 18:452-468

16. Wolska-Washer A, Robak P, Smolewski P, Robak T (2017) Emerging antibody-drug conjugates for treating lymphoid malignancies. Expert Opin Emerg Drugs 22:259-273
17. Mehta A, Forero-Torres A (2015) Development and integration of antibody-drug conjugate in non-Hodgkin lymphoma. Curr Oncol Rep 17:41

18. Morschhauser F, Flinn IW, Advani R, Sehn LH, Diefenbach C, Kolibaba K, Press OW, Salles G, Tilly H, Chen AI, Assouline S, Cheson BD, Dreyling M, Hagenbeek A, Zinzani PL, Jones S, Cheng J, Lu D, Penuel E, Hirata J, Wenger M, Chu YW, Sharman J (2019) Polatuzumab vedotin or pinatuzumab vedotin plus rituximab in patients with relapsed or refractory nonHodgkin lymphoma: final results from a phase 2 randomised study (ROMULUS). Lancet Haematol 6:e254-e265

19. Tilly H, Morschhauser F, Bartlett NL, Mehta A, Salles G, Haioun C, Munoz J, Chen Al, Kolibaba K, Lu D, Yan M, Penuel E, Hirata J, Lee C, Sharman JP (2019) Polatuzumab vedotin in combination with immunochemotherapy in patients with previously untreated diffuse large B-cell lymphoma: an open-label, non-randomised, phase 1b-2 study. Lancet Oncol 20:998-1010

20. Lucas AT, Price LSL, Schorzman AN, Storrie M, Piscitelli JA, Razo J, Zamboni WC (2018) Factors affecting the pharmacology of antibody-drug conjugates. Antibodies 7:10

21. Tolcher AW (2016) Antibody drug conjugates: lessons from 20 years of clinical experience. Ann Oncol 27:2168-2172

22. Perez HL, Cardarelli PM, Deshpande S, Gangwar S, Schroeder GM, Vite GD, Borzilleri RM (2014) Antibody-drug conjugates: current status and future directions. Drug Discov Today 19:869-881

23. Vezina HE, Cotreau M, Han TH, Gupta M (2017) Antibodydrug conjugates as cancer therapeutics: past, present, and future. J Clin Pharmacol 57:S11-S25

24. US Department of Health and Human Services, Food and Drug Administration, Center for Drug Evaluation and Research (2012) Guidance for industry, drug interaction studies-study design, data analysis, implications for dosing, and labeling recommendations. US Department of Health and Human Services, Silver Spring

25. European Medicines Agency, Committee for Human Medicinal Products (2015) Guideline on the investigation of drug interactions. 21 June 2012. European Medicines Agency, London

26. Kraynov E, Kamath AV, Walles M, Tarcsa E, Deslandes A, Iyer RA, Datta-Mannan A, Sriraman P, Bairlein M, Yang JJ, Barfield M, Xiao G, Escandon E, Wang W, Rock DA, Chemuturi NV, Moore DJ (2016) Current approaches for absorption, distribution, metabolism, and excretion characterization of antibody-drug conjugates: an industry white paper. Drug Metab Dispos 44:617-623

27. Han TH, Zhao B (2014) Absorption, distribution, metabolism, and excretion considerations for the development of antibody-drug conjugates. Drug Metab Dispos 42:1914-1920

28. Lu D, Sahasranaman S, Yi Z, Girish S (2013) Strategies to address drug interaction potential for antibody-drug conjugates in clinical development. Bioanalysis 5:1115-1130

29. Kaur S, Xu K, Saad OM, Dere RC, Carrasco-Triguero M (2013) Bioanalytical assay strategies for the development of antibodydrug conjugate biotherapeutics. Bioanalysis 5:201-226

30. Gorovits B, Alley SC, Billic S, Booth B, Kaur S, Oldfield P, Purushothama S, Rao C, Shord S, Siguenza P (2013) Bioanalysis of antibody-drug conjugates: American Association of Pharmaceutical Scientists Antibody-drug Conjugate Working Group position paper. Bioanalysis 5:997-1006

31. US Food and Drug Administration 2018 Bioanalytical method validation. https://www.fda.gov/files/drugs/published/Bioanalyti cal-Method-Validation-Guidance-for-Industry.pdf. Accessed 16 Jan 2020

32. US Food and Drug Administration 2015 Analytical procedures and method validation for drugs and biologics. https://www.fda. gov/files/drugs/published/Analytical-Procedures-and-MethodsValidation-for-Drugs-and-Biologics.pdf. Accessed 16 Jan 2020 
33. European Medicines Agency (2011) Guideline on bioanalytical method validation. https:/www.ema.europa.eu/en/documents/ scientific-guideline/guideline-bioanalytical-method-validation _en.pdf. Accessed 16 Jan 2020

34. Lu D, Lu T, Gibiansky L, Li X, Li C, Agarwal P, Shemesh CS, Shi R, Dere RC, Hirata J, Miles D, Chanu P, Girish S, Jin JY (2019) Integrated two-analyte population pharmacokinetic model of polatuzumab vedotin in patients with non-Hodgkin lymphoma. CPT Pharmacomet Syst Pharmacol. https://doi.org/10.1002/ psp4.12482 (Epub ahead of print)

35. Phillips T, Brunvand M, Chen A, Press O, Essell J, Chiappella A, Diefenbach C, Jones S, Hirata J, Flinn IW (2016) Polatuzumab vedotin combined with obinutuzumab for patients with relapsed or refractory non-Hodgkin lymphoma: preliminary safety and clinical activity of a Phase Ib/II study. Blood 128:622

36. Drake PM, Rabuka D (2017) Recent developments in ADC technology: preclinical studies signal future clinical trends. BioDrugs 31:521-531

37. Mukherjee A, Waters AK, Babic I, Nurmemmedov E, Glassy MC, Kesari S, Yenugonda VM (2019) Antibody drug conjugates: progress, pitfalls, and promises. Hum Antibodies 27:53-62

38. De Goeij BE, Lambert JM (2016) New developments for antibody-drug conjugate-based therapeutic approaches. Curr Opin Immunol 40:14-23

39. Mahmood I, Green MD (2007) Drug interaction studies of therapeutic proteins or monoclonal antibodies. J Clin Pharmacol 47:1540-1554

40. Prueksaritanont T, Chu X, Gibson C, Cui D, Yee KL, Ballard J, Cabalu T, Hochman J (2013) Drug-drug interaction studies: regulatory guidance and an industry perspective. AAPS J 15:629-645

41. Kenny JR, Liu MM, Chow AT, Earp JC, Evers R, Slatter JG, Wang DD, Zhang L, Zhou H (2013) Therapeutic protein drugdrug interactions: navigating the knowledge gaps-highlights from the 2012 AAPS NBC Roundtable and IQ Consortium/FDA Workshop. AAPS J 15:933-940

42. Kamath AV, Iyer S (2016) Challenges and advances in the assessment of the disposition of antibody-drug conjugates. Biopharm Drug Dispos 37:66-74
43. Chen Y, Samineni D, Mukadem S, Wong H, Shen BQ, Lu D, Girish S, Hop C, Jin JY, Li C (2015) Physiologically based pharmacokinetic modeling as a tool to predict drug interactions for antibody-drug conjugates. Clin Pharmacokinet 54:81-93

44. Olivier KJ, Hurvitz SA (2016) Antibody-drug conjugates fundamentals, drug development, and clinical outcomes to target cancer. Wiley, Hoboken

45. Lu D, Gillespie WR, Girish S, Agarwal P, Li C, Hirata J, Chu YW, Kagedal M, Leon L, Maiya V, Jin JY (2017) Time-to-event analysis of polatuzumab vedotin-induced peripheral neuropathy to assist in the comparison of clinical dosing regimens. CPT Pharmacomet Syst Pharmacol 6:401-408

46. Deslandes A (2014) Comparative clinical pharmacokinetics of antibody-drug conjugates in first-in-human Phase 1 studies. MAbs 6:859-870

47. Malik P, Phipps C, Edginton A, Blay J (2017) Pharmacokinetic considerations for antibody-drug conjugates against cancer. Pharm Res 34:2579-2595

48. Vitolo U, Trneny M, Belada D, Burke JM, Carella AM, Chua N, Abrisqueta P, Demeter J, Flinn I, Hong X, Kim WS, Pinto A, Shi YK, Tatsumi Y, Oestergaard MZ, Wenger M, FingerleRowson G, Catalani O, Nielsen T, Martelli M, Sehn LH (2017) Obinutuzumab or rituximab plus cyclophosphamide, doxorubicin, vincristine, and prednisone in previously untreated diffuse large B-cell lymphoma. J Clin Oncol 35:3529-3537

49. Lu D, Jin JY, Gibiansky L, Gillespie WR, Agarwal P, Jones C, Chu YW, Wenger MK, Hirata J, Li C, Girish S (2015) Exposureresponse analysis to assist selection of dose and treatment duration for polatuzumab vedotin as a single agent or in combination with rituximab for the treatment of B-cell lymphoma. Blood 126:1525

Publisher's Note Springer Nature remains neutral with regard to jurisdictional claims in published maps and institutional affiliations. 